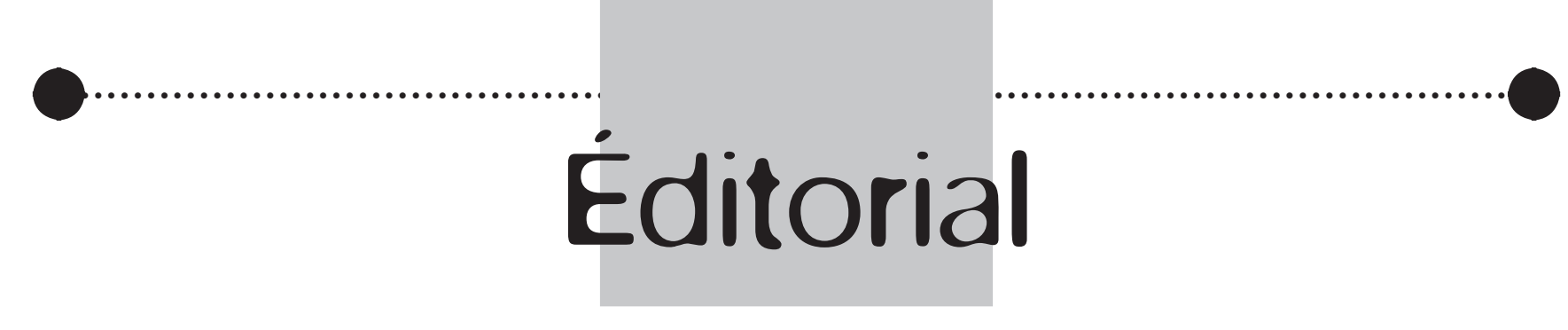

\title{
Une coupure qui nous relie autrement
}

\section{A break that connects us differently}

\author{
Michel WAWRZYNIAK
}

Rédacteur en chef

de Perspectives Psy

Psychologue Clinicien,

Psychanalyste

et Thérapeute Familial.

Professeur émérite en

Psychologie Clinique

à l'Université

de Picardie Jules Verne d'Amiens, 1 ,

chemin du Thil,

80000 Amiens, France. michel.wawrzyniak@ u-picardie.fr

michel.wawrzyniak@ wanadoo.fr

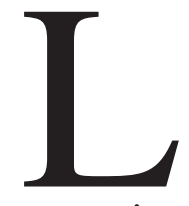

e symbole était, dans la Grèce archaïque, une pièce de céramique cassée en deux qui reliait deux camps alliés qui pouvaient ainsi reconnaître, grâce à elle, un porteur de message de l'autre camp. Le symbole était une coupure qui liait. Le symbole est une coupure qui lie.

La coupure suscitée par la pandémie et les mesures de confinement nous a éloignés les uns des autres mais elle nous a reliés autrement. Le téléphone et les appels sur Skype, Zoom, Teams... sont devenus les nouveaux canaux vitaux de nos liens humains.

La tenue de la dernière réunion du comité de rédaction via une visioconférence en aura été un exemple remarquable. Ainsi, avant de nous engager dans le traitement de l'ordre du jour concernant notre activité éditoriale en cours et à venir, chacun d'entre nous a pu évoquer sa situation personnelle et professionnelle, la réalité pesante de la Covid-19 nous marquant tous. Le fait que chacun d'entre nous soit impliqué dans des situations cliniques et institutionnelles souvent très différentes nous a offert un large panorama, à la fois vaste et très précis, des effets de la pan- démie sur nos proches, nos patients, nos étudiants, nos collègues de très nombreuses professions, et nos pratiques, que ce soit au SAMU, dans les CUMP, les services de psychiatrie, d'addictologie, les CHU, les hôpitaux généraux, les services d'urgence, des numéros vert de soutien en ligne à la parentalité en confinement, des services d'aide aux familles d'accueil, la continuité pédagogique dans les universités et centres de formations, en activité libérale...

Certains d'entre nous, touchés par le virus, participant malgré tout à notre réunion. D'autres ne le pouvant pas. Un moment exceptionnel de fraternité partagée entre nous mais aussi témoignant de nos proximités psychiques avec beaucoup d'autres. Un moment mémorable duquel a jailli, comme une évidente nécessité, le projet de témoigner de toutes ces situations, de rendre publiques et partagées nos expériences et nos réflexions dans un numéro à venir de Perspectives Psy: épidémies et psychiatrie, dossier qui sera mené sous la direction de Jacques Fortineau et de Brice Martin.

La pandémie nous a rudement frappés. Elle a rudement frappé nos proches, 
nos collègues, nos patients. À quoi tiennent nos vies ? A-t-on souvent entendu s'interroger nos interlocuteurs. Au mince fil de la protection de la distanciation physique? Une distanciation que nos statuts de soignants n'ont pas toujours pu nous garantir. Dans le champ de la psychiatrie, de nombreux témoignages ont exprimé l'oubli chronique de la psychiatrie par les politiques $^{1}$ ou encore l'insuffisance en moyens matériels de protection au point que bien des collectifs ont exprimé leur colère d'être ainsi exposés à des risques dramatiquement sous-estimés, politiquement mal évalués, aux premiers temps de la pandémie ${ }^{2}$.

Si le symbole réunit deux pièces de céramique dans l'espace, il réunit aussi, dans le temps, les deux moments entre un événement et son analyse. Viendra le temps de dire ce qui s'est vraiment passé et de tenir compte de ce à quoi nous avons été voués, une fois la crise pandémique passée. La traversée de la terra incognita ${ }^{3}$ de la pandémie ressemblera-t-elle à toute autre traversée de crise : par-delà notre vécu d'angoisse et d'incertitude aurons-nous la possibilité de créer des liens et des manières de penser et de faire nouveaux ? La pandémie nous a bousculés dans la réalisation de ces deux premiers numéros de l'année de Perspectives Psy. Nous avons déployé tous nos efforts pour destiner rapidement successivement à nos lecteurs les deux premiers numéros de notre revue correspondant au premier semestre de l'année 2020, rédigés dans un même élan de concep-

1. «Psychiatrie oubliée ? ", Éditorial d'avril $2020 \mathrm{du}$ Dr Michel David, président de la Fédération Française de Psychiatrie.

2. «On a été mis en danger» : à Neuilly-sur-Marne, la détresse des soignants d'un hôpital psychiatrique, BFMTV, 02.04.2020.

3. «Le temps de l'incertitude», Douglas Kennedy in Le Monde des 31 mai-1 $1^{\text {er }}$ juin 2020, p. 27. tion. D'où cet éditorial présentant ces deux numéros ensemble.

Dans le $n^{\circ} 1 / 2020$, Pierre Delion nous a offert un éditorial, « Le moment Tosquelles », qui parle au plus près de ce que nous vivons aujourd'hui rapporté à ce que la psychiatrie des dernières décennies a eu à vivre, a eu à subir, mais aussi a su créer. «Il va donc falloir réfléchir "pour de bon" au soir de cette période noire, et entreprendre ce partage nécessaire dans la conduite des affaires du monde : les personnes et les choses ne sont pas réductibles les unes aux autres », pour reprendre Pierre Delion dans son éditorial. Un de nos deux dossiers témoigne ensuite des réflexions et des propositions d'un groupe de travail mis en place par la Fédération Française de Psychiatrie (FFP), d'abord mené par Danièle Roche-Rabreau puis par Patrick Bantman consacré à la question du «Travail avec la famille lors de l'hospitalisation pour un premier épisode psychotique $»$. La FFP a institué ce groupe de travail à la suite de la bouleversante lettre que lui a adressée un père de patient. Une seconde partie de ce dossier viendra le compléter dans un de nos numéros à venir. Ce dossier nous invite ainsi à reprendre la réflexion de Henri Ey qui, à propos des épisodes psychotiques aigus chez de jeunes gens, posait cette question vertigineuse: «Folie d'un moment ou début de la folie de toute une existence ». Avec ici la mise en exergue du questionnement corollaire: "Quelle place de la famille dans une telle situation? ». L'autre dossier déploie une réflexion relative à la Psychiatrie de la Personne Âgée nous invitant à maintenir tendue notre vigilance sur les âges avancés. Le Dr Georges Jovelet aura coordonné ce dossier avec beaucoup de détermination.

Le numéro $2 / 2020$ est porteur, quant à lui, d'un dossier qui rend compte d'un 
colloque franco-canadien de psychiatrie et de psychopathologie qui s'est tenu à Montréal, en juillet 2019, organisé par le Centre de recherche et d'intervention sur le suicide, enjeux éthiques et pratiques de fin de vie (CRISE) dirigé par le Pr Réal Labelle et le Groupe d'étude de psychiatrie, psychologie et sciences sociales (GEPPSS), représenté par le Dr Hervé Benhamou, rigoureux coordonnateur de ce dossier avec Réal Labelle. Ce colloque avait pour thème : "Dépression et conduites suicidaires à l'adolescence ».

Notre ami, le professeur Réal Labelle, est une figure bien connue en France et dans la francophonie. Il nous a fait, à de nombreuses reprises, la faveur de sa présence et de ses interventions dans les congrès organisés sur le territoire national, par exemple, celui la SFPEADA en 2017 ou de ses prestations de formation auprès de jeunes psychiatres et psychologues français, entre autres, à l'université de Picardie Jules Verne d'Amiens.

Comme lui, son ami, le Pr Jean-Marc Guilé, qui a été rédacteur en chef de Perspectives Psy pendant sept années, connaît très bien les deux rives de l'Atlantique. Un des moments forts de ce colloque de l'été 2019 aura été d'accompagner Jean-Marc Guilé dans ses retrouvailles avec ses collègues de l'Université McGill à l'occasion de la visite du dispositif de soins pour adolescents déprimés à l'Institut Douglas. Un moment riche en émotions témoignant de l'hospitalité si généreuse qui nous aura été offerte par l'UQAM. C'est autour de nos collègues canadiens hautement spécialisés en études de suicidologie au Canada que nous avons tenu ce séminaire fermé, d'une trentaine de participants, mais ouvert sur la promesse de construire de nouvelles étapes permettant la poursuite de nos échanges devenant davantage accessibles à un public élargi. Ce dossier constitue donc une première étape de cet élargissement espéré. les contributions des collègues français participant à ce colloque franco-canadien seront publiés dans un numéro suivant.

Dans les contacts amicaux informels que nous avons eus durant ce séjour, nous nous souvenons de notre conversation avec Alain Lesage, épidémiologiste mondialement réputé, que nous avions déjà croisé lors du congrès de l'IACAPAP à Durban, en 2014, évoquant sa fascination pour l'ouvrage d'Albert Camus La peste... Un échange anté-pandémique, rétroactivement troublant et vertigineux par sa dimension prémonitoire. Nous savons que, depuis, parmi les villes nord-américaines Montréal, comme New York, a été une des plus touchées par la pandémie. Merci à nos collègues canadiens d'avoir réussi, malgré tout, à nous envoyer leurs contributions.

Dans ces deux numéros, 1 et $2 / 2020$, plusieurs articles originaux retiendront, eux aussi, l'intérêt de nos lecteurs.

Notamment celui Pierre Delion qui nous a accordé un entretien à la suite de l'offre faite de son éditorial. Cet entretien nous a fait nous approcher du code existentiel de la trajectoire d'un psychiatre ancré dans une psychopathologie transférentielle, porteur de valeurs humanistes et toujours ouvert aux apports de la science dans la créativité de la co-construction en équipe, en institution, insistant d'une manière vitale sur la dimension politique du métier de psychiatre. Cet entretien constitue un rebond très heureux de l'analyse de son livre Mon combat pour une psychiatrie humaine que la revue avait donnée il y trois ans.

Si le symbole réunit deux pièces de céramique dans l'espace, il réunit aussi, dans le temps, les deux moments entre 
un vœu que l'on formule et sa réalisation.

Alors nous souhaitons que ces deux numéros, qui parcourent, autant qu'ils le peuvent, l'espace et le temps, l'histoire et de la géographie de la psychopathologie, des soins en psychiatrie et des sciences humaines et sociales, nous in- vitent à persévérer, en tant que revue, dans notre effort tendu d'y être présents et agissants avec les plus vives de nos capacités de symbolisation.

\section{LIENS D'INTÉRÊT}

L'auteur déclare n'avoir aucun lien d'intérêt concernant les données publiées dans cet article.

\section{Communiqué \\ Communiqué de Presse du Collège Pédopsychiatrie de la FFP, le 20 mai 2020}

\section{Pour une pédopsychiatrie publique au service de tous}

La crise sans précédent liée à l'épidémie de Covid-19 a affecté nombre d'enfants et de familles pendant le confinement. L'après-confinement va malheureusement révéler son lot de troubles psychiques graves. Dans le service public de pédopsychiatrie, les équipes pluri-professionnelles des CMP (Centres MédicoPsychologiques) ont organisé les soins en urgence et assuré leur continuité sous diverses modalités : maintien de consultations présentielles, réunions et groupes thérapeutiques virtuels, outils de guidance envoyés aux familles, soins sur le lieu de vie, à domicile ou dans le quartier, chaque fois que nécessaire. Le travail de consultation à distance par téléphone ou visio-consultation, peu utilisé auparavant, s'est intensivement déployé. Nous avons prévenu et endigué des crises graves là où les troubles étaient majorés par le confinement. Nous nous sommes appuyés sur les liens tissés avec l'enfant et sa famille et sur le maillage partenarial local antérieur (scolaire, socio-éducatif et judiciaire, médico-social,professionnels libéraux...). Nous avons répondu aux difficultés de l'enfant et aux bouleversements du système familial ainsi qu'aux questionnements des familles, suivies ou non, sur le vécu des enfants. La méthode des pratiques intégratives propre à la pédopsychiatrie a facilité les interventions préventives et thérapeutiques : elle prend en compte l'enfant dans sa complexité développementale au sein de sonenvironnement.

La réactivité de ce dispositif montre le bien-fondé des unités sectorielles de proximité proposant accueil et soins, sans exclusive, quelle que soit la catégorie diagnostique qui les concerne, pour tous les enfants et adolescents y compris les plus vulnérables. La disponibilité de ces unités de proximité montre, a contrario, les limites de la multiplication des dispositifs de diagnostic et d'évaluation, où les enfants sont appréhendés à travers un trouble spécifique, qui ne s'engagent pas au-delà de préconisations d'interventions circonscrites et qui sont inadaptés aux situations complexes, de vulnérabilité ou de crise.

L'épidémie de Covid survient dans un contexte de discrédit envers la pédopsychiatrie publique et de réduction constante des budgets qui lui sont accordés. Pourtant cette période a fait la preuve de la pertinence et de l'engagement des équipes soignantes et a pointé l'inconséquence des décisions en matière d'offre sanitaire.

Les secteurs de pédopsychiatrie reprennent le cours habituel des soins, marqués par cette douloureuse expérience, qui a mis en exergue l'impact psychique de l'isolement des enfants, adolescents et de leurs familles, des violences intra-familiales, de l'aggravation de la précarité sociale et du décrochage scolaire. Les possibilités offertes par les nouvelles technologies déployées durant la crise ne pourront pas remplacerle lien social, éducatif et thérapeutique inhérent aux pratiques intégratives. La diversité des modalités d'action sera à financer dans une nouvelle approche budgétaire, à construire en fonction de missions globales. Les souffrances psychiques liées aux traumatismes psychiques multiples des adultes et de leurs enfants en lien avec la pandémie Covid-19 ne pourront être prises en charge par la pédopsychiatrie que dans la mesure où ses structures (notamment ses CMP) seront soutenues financièrement et non disqualifiées dans leur exercice!

Dès lors, il est urgent de soutenir un service public de qualité en pédopsychiatrie, afin qu'il soit accessible à tous, et de définir une véritable politique globale de santé répondant à lènsemble des besoins.

Renseignements

La Fédération Française de Psychiatrie regroupe les associations scientifiques et syndicats de psychiatres Secrétariat : IPB Sainte-Anne - 26, boulevard Brune - 75014 PARIS - Tél. : 0148047341 contact@fedepsychiatrie.fr - www.fedepsychiatrie.fr 